\title{
Proof Methodologies for Behavioural Equivalence in DPI
}

\author{
Alberto Ciaffaglione ${ }^{1,2}$, Matthew Hennessy ${ }^{2}$, and Julian Rathke ${ }^{2}$ \\ 1 Dipartimento di Matematica e Informatica, Università di Udine, Italia \\ ciaffagl@dimi.uniud.it \\ 2 Department of Informatics, University of Sussex, United Kingdom \\ \{A.Ciaffaglione, M.Hennessy, J.Rathke\}@sussex.ac.uk
}

\begin{abstract}
We focus on techniques for proving behavioural equivalence between systems in DPI, a distributed version of the PICALCulus in which processes may migrate between dynamically created locations, and where resource access policies are implemented by means of capability types.

We devise a tractable collection of auxiliary proof methods, relying mainly on the use of bisimulations up-to $\beta$-reductions, which considerably relieve the burden of exhibiting witness bisimulations. Using such methods we model simple distributed protocols, such as crossing a firewall, a server and its clients, metaservers installing memory services, and address their correctness in a relatively simple manner.
\end{abstract}

\section{Introduction}

Bisimulations [Mil89], and the related bisimulation equivalence, have been proved to be of central importance in the elaboration of semantic theories of processes, and in developing verification techniques for them. The purpose of this work is to demonstrate that they may be also employed for the verification of distributed systems, even when the correctness depends on access control policies.

We focus on an abstract system description language called DPI [HR02b], an extension of the well-known PICALCULUs [MPW92,SW01]. In this language a system consists of a collection of processes, or agents, distributed among different sites, where they can use local resources; these resources are modelled using local versions of PICALCULus communication channels. Agents may migrate from site to site, generate new local resources, or indeed new sites.

Following ideas originally formulated in [PS00], DPI can be endowed with a system of capability types, with which access policies to both resources and sites can be expressed. Since the behaviour of systems is dependent on the access policy in force, a new theory of semantic equivalence is required to take this dependency into account. This was developed in [HR02a,HMR04], where the equivalence is expressed in the form of triples

$$
\mathcal{I} \models M \approx_{b i s} N
$$

Intuitively this means that the systems $M$ and $N$ exhibit the same behaviour, from the point of view of a user constrained by the access policy $\mathcal{I}$; formally, $\mathcal{I}$ is simply a type environment, giving, for each resource and location, the capabilities which may be exercised by the user.

In this paper we show that this relativised notion of system behaviour can be effectively employed to demonstrate the correctness of access protocols for distributed 
Base Types: $\quad$ base $::=$ int $\mid$ bool $\mid$ unit $|\mathrm{T}| \ldots$

Value Types: $\quad A::=$ base $|\mathrm{C}| \mathrm{C}_{\circledast}$ loc $\mid \mathrm{K}$

Local Channel types: $C::=r\langle T\rangle|w\langle T\rangle| r w\langle T\rangle$

Location Types: $\quad \mathrm{K}::=\operatorname{loc}\left[c_{1}: \mathrm{C}_{1}, \ldots, c_{n}: \mathrm{C}_{n}\right], n \geq 0$ (provided $c_{i}=c_{j}$ implies $i=j$ )

Transmission Types: $\mathrm{T}::=\left(\mathrm{A}_{1}, \ldots, \mathrm{A}_{n}\right), n \geq 0$

Fig. 2. Types for DPI - informal

use are given in Figure 2. Apart from the standard base types, and the special top type $T$, the main ones are

local channel types: these are ranged over by $\mathrm{C}$ and can take the form $\mathrm{rw}\langle\mathrm{T}\rangle$, giving the ability to both read and write values of type $T$, or the restricted supertypes $r\langle T\rangle$ and $\mathrm{w}\langle\mathrm{T}\rangle$;

non-local channel types: these take the form $\mathrm{C}_{\circledast}$ loc, and a value of this type is a structured value, $c @ l$;

location types: these take the form $\operatorname{loc}\left[c_{1}: \mathrm{C}_{1}, \ldots, c_{n}: \mathrm{C}_{n}\right]$; receiving a value $l$ of this type gives access to the channels, or resources, $c_{i}$ at type $\mathrm{C}_{i}$, for $1 \leq i \leq n$.

The types come equipped with a subtyping relation, which is defined inductively, from the standard requirements on channel types, and record subtyping on location types $\operatorname{loc}\left[c_{1}: \mathrm{C}_{1}, \ldots c_{n}: \mathrm{C}_{n}\right]<: \operatorname{loc}\left[c_{1}: \mathrm{C}_{1}, \ldots c_{k}: \mathrm{C}_{k}\right]$, whenever $k \leq n$. Viewing types (intuitively) as sets of capabilities, $T_{1}<: T_{2}$ means that the capabilities of $T_{2}$ are a subset of those of $T_{1}$.

The static typing of a system, $\Gamma \vdash M$, indicates that $M$ uses all its identifiers in accordance with the types designated in the type environment $\Gamma$, which gives the type of all the free names in $M$. Formally, a type environment $\Gamma$ is a consistent list of entries, which must take one of the following forms

- $u$ : loc, indicating $u$ is to be used as a location;

- $u @ w:$ C, indicating that $w$ is already known to $\Gamma$ as a location, and $u$ is a local channel at $w$ with type $\mathrm{C}$.

The typing of systems requires an auxiliary typing judgment for agents, $\Gamma \vdash_{k} P$, which needs to be parameterised relative to the current location $k$, because resources are located: they may be available at one site and not another.

Behaviour. The behaviour of a system, that is the ability of its agents to interact with other agents, depends on the knowledge these agents have of each other capabilities.

Definition 1 (Configurations). A configuration consists of a pair $\mathcal{I} \triangleright M$, where

- I is a type environment which associates some type to every free name in $M$

- there is a type environment $\Gamma$ such that $\Gamma \vdash M$ and $\Gamma<: \mathcal{I}$

This latter requirement means that if $\mathcal{I}$ can assign a type $\mathrm{T}_{\mathcal{I}}$ to a name $n$, then $\Gamma$ can assign a type $\mathrm{T}_{\Gamma}$ such that $\mathrm{T}_{\Gamma}<: \mathrm{T}_{\mathcal{I}}$. Again, viewing types as sets of capabilities, this means that $\mathrm{T}_{\mathcal{I}}$, representing the knowledge of the external user, is a subset of $\mathrm{T}_{\Gamma}$, the actual set of capabilities used to type the system $M$. 


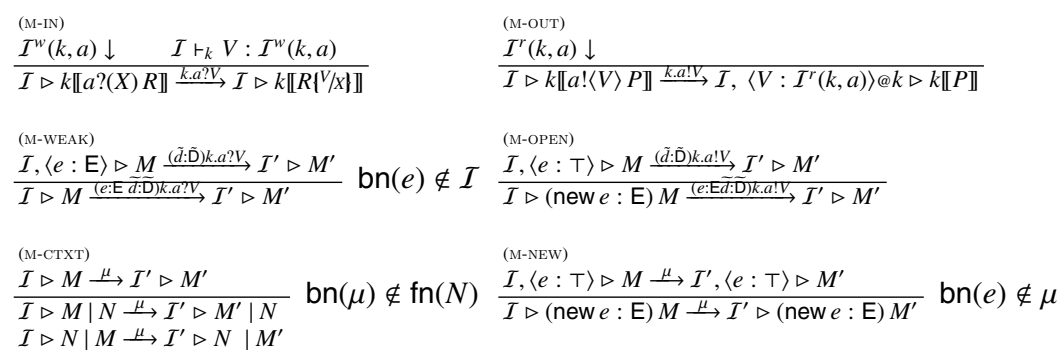

Fig. 3. External actions-in-context for DPI

\begin{tabular}{|c|c|}
\hline $\begin{array}{l}\text { (M-COMM) } \\
\mathcal{I}_{1} \triangleright M \stackrel{(\tilde{e}: \tilde{E}) k \cdot a ? V}{\longrightarrow} I_{1}^{\prime} \triangleright M^{\prime} \\
\mathcal{I}_{2} \triangleright N \stackrel{(\tilde{e}: \tilde{E}) k \cdot a ! V}{\longrightarrow} I_{2}^{\prime} \triangleright N^{\prime} \\
\end{array}$ & $\begin{array}{l}\text { (м-сомм) } \\
\mathcal{I}_{1} \triangleright M \stackrel{(\tilde{e}: \tilde{E}) k \cdot a ! V}{\longrightarrow} I_{1}^{\prime} \triangleright M^{\prime} \\
\mathcal{I}_{2} \triangleright N \stackrel{(\tilde{e}: \tilde{E}) k \cdot a ? V}{\longrightarrow} \mathcal{I}_{2}^{\prime} \triangleright N^{\prime} \\
\end{array}$ \\
\hline 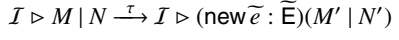 & $\overline{\mathcal{I} \triangleright M \mid N \stackrel{\tau}{\longrightarrow} \mathcal{I} \triangleright(\text { new } \widetilde{e}: \widetilde{\mathrm{E}})\left(M^{\prime} \mid N^{\prime}\right)}$ \\
\hline$\stackrel{\text { (M-MOVE) }}{\mathcal{I} \triangleright k \llbracket \text { goto } l . P \rrbracket \stackrel{\tau}{\longrightarrow} \beta} \mathcal{I} \triangleright l \llbracket P \rrbracket$ & $\begin{array}{lll}\text { (M-EQ/NEQ) } & \\
\mathcal{I} \triangleright k \llbracket \text { if } v_{1}=v_{2} \text { then } P \text { else } Q \rrbracket \stackrel{\tau}{\longrightarrow} \beta \mathcal{I} \triangleright k \llbracket P \rrbracket \quad\left(v_{1}=v_{2}\right) \\
\mathcal{I} \triangleright k \llbracket \text { if } v_{1}=v_{2} \text { then } P \text { else } Q \rrbracket \stackrel{\tau}{\longrightarrow}_{\beta} \mathcal{I} \triangleright k \llbracket Q \rrbracket \quad\left(v_{1} \neq v_{2}\right)\end{array}$ \\
\hline 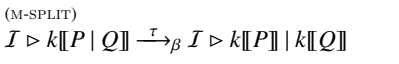 & $\begin{array}{l}\text { (M-L.CREATE) } \\
\mathcal{I} \triangleright k \llbracket(\text { newloc } l: \mathrm{L}) P \rrbracket \\
\stackrel{\tau}{\longrightarrow}_{\beta} \mathcal{I} \triangleright(\text { new } l: \mathrm{L}) k \llbracket P \rrbracket\end{array}$ \\
\hline$\stackrel{\text { (M-UNwIND) }}{\mathcal{I} \triangleright k \llbracket * P \rrbracket} \stackrel{\tau}{\longrightarrow}_{\beta} \mathcal{I} \triangleright k \llbracket * P \mid P \rrbracket$ & 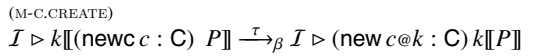 \\
\hline
\end{tabular}

Fig. 4. Internal actions-in-context for DPI

So we define the behaviour in terms of actions over configurations, that is

$$
\mathcal{I} \triangleright M \stackrel{\mu}{\longrightarrow} \mathcal{I}^{\prime} \triangleright M^{\prime}
$$

where the label $\mu$ can take any of the following forms

- $\tau$ : an internal action, requiring no participation by the user;

- ( $\tilde{e}: \tilde{E}) k \cdot a$ ? $V$ : the input of value $V$ along the channel $a$, located at the site $k$. The bound names in $(\tilde{e})$ are freshly generated by the user;

- ( $\tilde{e}: \tilde{E}) k \cdot a ! V:$ the output of value $V$ along the channel $a$, located at the site $k$. The bound names in $(\tilde{e})$ are freshly generated by the environment.

The rules for defining these actions are given in Figure 3 and Figure 4, a slightly different but equivalent formulation to that given in [HMR04]. The guiding principle for (1) to happen, is that $M$ must be able to perform the action $\mu$, and the user must have, in $\mathcal{I}$, the capability to participate in the action. The rules use some new notation for looking up the types associated with channels in environments: the partial functions $\mathcal{I}^{r}(k, a)$ and $\mathcal{I}^{w}(k, a)$ return the read, respectively write, type associated with the channel $a$ at the location $k$ in $\mathcal{I}$ (of course these may not exist, and $\mathcal{I}^{w}(k, a) \downarrow$, for example, indicates that the write type is indeed defined). We extract names from entries in environments with the function bn(-), defined by $\operatorname{bn}(u)=u$ and $\operatorname{bn}(u @ w)=u$; this is extended to actions $\mu$ in the obvious manner. Notice also that we use the notation $\mathrm{fn}(-)$ for free variables. Finally, we have labelled some internal actions in Figure 4 as $\beta$-actions, which will be useful in the next section; but for the moment these labels can be ignored.

We now have a labelled transition system in which the states are configurations, and we can apply the standard definition of (weak) bisimulation. 
(S-EXTR)

$(\mathrm{S}-\mathrm{COM})$

(S-ASSOC)

(S-ZERO)

$$
(\text { new } e: \mathrm{E})(M \mid N) \equiv M \mid(\text { new } e: \mathrm{E}) N \text {, if } \operatorname{bn}(e) \notin \mathrm{fn}(M)
$$

$M|N \equiv N| M$

$(M \mid N)|O \equiv M|(N \mid O)$

$M \mid \mathbf{0} \equiv M$

$k \llbracket$ stop $\rrbracket=0$

$(\mathrm{S}-\mathrm{FLIP}) \quad($ new $e: \mathrm{E})\left(\right.$ new $\left.e^{\prime}: \mathrm{E}^{\prime}\right) M \equiv\left(\right.$ new $\left.e^{\prime}: \mathrm{E}^{\prime}\right)($ new $e: \mathrm{E}) M$, if $\operatorname{bn}(e) \notin\left(e^{\prime}: \mathrm{E}^{\prime}\right), \operatorname{bn}\left(e^{\prime}\right) \notin(e: \mathrm{E})$

Fig. 5. Structural equivalence for DPI

Definition 2 (Bisimulations). We say a binary relation over configurations is a bisimulation if both it, and its inverse, satisfy the following transfer property

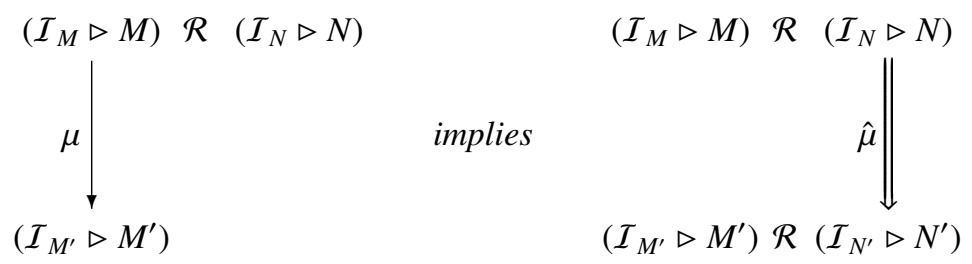

Here we use standard notation, see [MPW92], with $\stackrel{\mu}{\longrightarrow}$ representing $\stackrel{\tau}{\longrightarrow} * \stackrel{\mu}{\longrightarrow} \circ \stackrel{\tau}{\longrightarrow}$, and $\stackrel{\hat{\mu}}{\Longrightarrow}$ meaning $\stackrel{\tau}{\longrightarrow}$, if $\mu$ is $\tau$, and $\stackrel{\mu}{\Longrightarrow}$ otherwise. This allows a single internal move to be matched by zero or more internal moves.

We let $\approx_{\text {bis }}$ denote the largest bisimulation between configurations.

Rather than writing $(\mathcal{I} \triangleright M) \approx_{b i s}(\mathcal{I} \triangleright N)$, we use the more suggestive notation $\mathcal{I} \vDash$ $M \approx_{b i s} N$. This can be viewed as a relation between systems, parameterised over type environments which represent user's knowledge of system's capabilities.

It is this bisimilarity $\approx_{b i s}$ which is the object of our study: we aim to show that, despite the complexity of its definition, tractable proof techniques can be developed for it. Finally, we should remark this is not an arbitrarily chosen version of bisimulation equivalence; in [HMR04] its definition is justified in detail: it is shown to be, in some sense, the largest reasonable typed equivalence between Dpi systems.

\section{Proof Techniques}

The basic method for showing that two systems $M$ and $N$ are equivalent, relative to an environment $\mathcal{I}$, is to exhibit a parameterised relation $\mathcal{R}$ such that $\mathcal{I} \vDash M \mathcal{R} N$, and demonstrate that it satisfies the requirements of being a bisimulation. In this section we give a number of auxiliary methods, which can considerably relieve the burden of exhibiting such relations. The following Theorem is proved in [HMR04], and justifies a form of contextual reasoning.

\section{Theorem 1 (Contextuality).}

- $\mathcal{I} \vDash M \approx_{b i s} N$ and $\mathcal{I} \vdash O$ imply $\mathcal{I} \vDash M\left|O \approx_{b i s} N\right| O$

- $\mathcal{I},\langle e: \mathrm{E}\rangle \vDash M \approx_{b i s} N$ implies $\mathcal{I} \vDash($ new $e: \mathrm{E}) M \approx_{b i s}($ new $e: \mathrm{E}) N$

We can also manipulate system descriptions. Let $\equiv$ be the least equivalence relation which satisfies the rules in Figure 5, and is preserved by the constructs $-\mid-$ and (new $e: \mathrm{E})(-)$; this is referred to as structural equivalence. 
Proposition 1. $M \equiv N$ implies $M \approx_{b i s} N$.

This means that we can employ the axioms in Figure 5 as equations for manipulations of systems preserving the semantics. For example, we can omit the termination process stop, because $k \llbracket$ stop $\rrbracket \equiv \mathbf{0}$ and $M \mid \mathbf{0} \equiv M$.

Further equations can be obtained by considering the internal actions in Figure 4. First observe that these actions do not change the environment of a configuration, and therefore, for convenience, let us abbreviate $\mathcal{I} \triangleright M \stackrel{\tau}{\longrightarrow} I \triangleright M^{\prime}$ to the simpler $\mathcal{I} \triangleright M \stackrel{\tau}{\longrightarrow} M^{\prime}$.

Proposition 2. $\mathcal{I} \triangleright M \stackrel{\tau}{\longrightarrow}{ }_{\beta}^{*} N$ implies $\mathcal{I} \vDash M \approx_{b i s} N$.

This Proposition gives more valid equations for reasoning about systems. Typical examples, obtained just by examining those axioms in Figure 4 which describe $\beta$-actions, include $k \llbracket P\left|Q \rrbracket \approx_{b i s} k \llbracket P \rrbracket\right| k \llbracket Q \rrbracket$ and $k \llbracket$ goto $l . P \rrbracket \approx_{b i s} l \llbracket P \rrbracket$ and $k \llbracket($ newc $c: \mathrm{C}) P \rrbracket \approx_{b i s}$ (new $c \Subset k: \mathrm{C}) k \llbracket P \rrbracket$.

But these $\beta$-labelled internal actions also provide us with a very powerful method for approximating bisimulations.

Definition 3 (Bisimulations up-to- $\beta$ ). A binary relation between configurations is said to be a bisimulation up-to- $\beta$ if it, and its inverse, satisfy the following transfer property
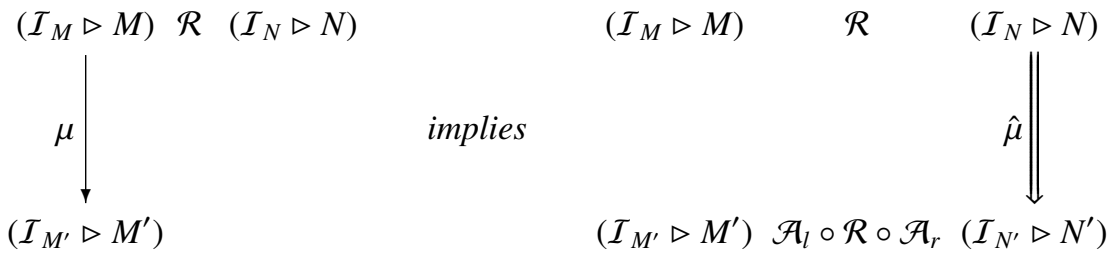

where $\mathcal{A}_{l}$ is the relation $\left(\sim_{\beta}^{*} \circ \equiv\right)$, and $\mathcal{A}_{r}$ is $\approx_{\text {bis }}$; strictly speaking, these relations are over systems, but they are lifted in the obvious manner to configurations.

The idea of these approximate bisimulations is that to match an action $\left(\mathcal{I}_{M} \triangleright M\right) \stackrel{\mu}{\longrightarrow}$ $\left(\mathcal{I}_{M^{\prime}} \triangleright M^{\prime}\right)$ it is sufficient to find a $\beta$-derivative of the residual $\left(\mathcal{I}_{M^{\prime}} \triangleright M^{\prime}\right) \stackrel{\tau}{\longrightarrow}_{\beta}^{*}\left(M^{\prime \prime}\right)$ and a matching action $\left(\mathcal{I}_{N} \triangleright N\right) \stackrel{\hat{\mu}}{\Longrightarrow}\left(\mathcal{I}_{N^{\prime}} \triangleright N^{\prime}\right)$ such that, up-to structural equivalence and bisimilarity, respectively, the pairs $\left(I_{M^{\prime}} \triangleright M^{\prime \prime}\right)$ and $\left(\mathcal{I}_{N^{\prime}} \triangleright N^{\prime}\right)$ are once more related. Intuitively, a configuration can represent all the configurations to which it can evolve using $\beta$-moves.

Proposition 3. If $(\mathcal{I} \triangleright M) \mathcal{R}(I \triangleright N)$, $\mathcal{R}$ a bisimulation up-to- $\beta$, then $\mathcal{I} \vDash M \approx_{b i s} N$.

\section{Crossing a Firewall}

Let us consider the firewall example, first proposed in [CG98] and studied at length in [GC99,LS00,MN03] within versions of Mobile Ambients. Intuitively, a firewall is a domain to which access is restricted: only agents which are permitted, in some sense, by the firewall, are allowed in. A simple example takes the form

$$
F \Leftarrow(\text { new } f: \text { F) } f \llbracket P \mid * \text { goto } a \text {.tell! }\langle f\rangle \rrbracket
$$


Here $f$ is the name of the firewall, which is created with the capabilities described in the location type $\mathrm{F}$, and $P$ is some code which maintains the internal business of the firewall. A typical example of the capabilities could be given by $F=\operatorname{loc}[i n f o$ : $r w\langle I\rangle, r e q: r w\langle R\rangle]$, which allow reading to and writing from two resources info and req in $f$. Then $P$ could, for example, maintain appropriate services at the resources; of course, it would also be able to use non-local resources it knows about in its current environment.

The existence of the firewall is made known only to another domain, $a$, via the information channel tell located there. An example is the following

$$
A \Leftarrow a \llbracket R \mid \text { tell?(x) goto } x . Q \rrbracket
$$

where $a$ is informed of $f$ by inputing on the local channel tell. If we consider an arbitrary type environment $\Gamma$, we have the execution

$$
\Gamma \triangleright F \mid A \stackrel{\tau}{\longrightarrow} \text { (new } f: \mathrm{F})(f \llbracket P \mid * \text { goto } a . \text { tell } !\langle f\rangle \mid Q \rrbracket) \mid a \llbracket R \rrbracket
$$

so the code $Q$ is allowed to execute locally within the firewall.

Notice that the resources to which $Q$ has access within the firewall are controlled by the capability type associated with the information channel tell. For example, suppose in $\Gamma$ the type associated with this channel is $r w\left\langle\mathrm{~F}_{r}\right\rangle$, where $\mathrm{F}_{r}=\operatorname{loc}[$ info : w $\langle\mathrm{I}\rangle$, req : $\mathrm{r}\langle\mathrm{R}\rangle]$. Then $\mathrm{F}_{r}$ is a supertype of the declaration type $\mathrm{F}$ : hence in (2), $Q$, having gained entry into the firewall, can only write to resource info and read from req.

Let us now consider the correctness of this simple protocol, which allows access of one agent, $Q$, to the firewall. Let $\Gamma$ be any type environment such that

$$
\Gamma \vdash F \mid A
$$

Then one might expect to be able to derive

$$
\Gamma \vDash F \mid A \approx_{b i s}(\text { new } f: \mathrm{F})(f \llbracket P \mid * \text { goto } a \text {.tell! }\langle f\rangle \mid Q \rrbracket) \mid a \llbracket R \rrbracket
$$

But this happens not to be true, because of the implicit assumption that the information channel tell in $a$ can only be accessed by partners in the entry protocol, $f$ and $a$. But, in order for (3) to be true, we must have $\Gamma \vdash_{a}$ tell : rw $\left\langle\mathrm{F}_{r}\right\rangle$, and this allows other agents in the environment access to tell. For example, consider

$$
\text { Rogue } \Leftarrow b \text { «goto } a \text {.tell! }\langle b\rangle \rrbracket
$$

and suppose that the only type inference from $\Gamma$ involving $b$ is $\Gamma \vdash b$ : loc; so $\Gamma$ is not aware of any resources at $b$. Nevertheless $\Gamma$ ト Rogue, and therefore Contextuality (Theorem 1) applied to (4) would give

$$
\Gamma \models F|A| \text { Rogue } \approx_{b i s}(\text { new } f: \mathrm{F})(f \llbracket P \mid * \text { goto } a \text {.tell! }\langle f\rangle \mid Q \rrbracket)|a \llbracket R \rrbracket| \text { Rogue }
$$

But this is obviously not the case, as the left-hand system can reduce via a series of $\tau$-steps (representing the interaction between $A$ and Rogue) to the state

$$
\Gamma \triangleright F|a \llbracket R \rrbracket| b \llbracket Q \rrbracket
$$


Under reasonable assumptions about the code $Q$, the right-hand system has no corresponding reduction to a similar state. On the left-hand side the code $Q$, now located at $b$, can not run, while on the right-hand side, no matter what $\tau$-steps are made, $Q$ will be able to execute at $f$. Thus (4) can not be true.

However, our framework allows us to amend the correctness statement (4) above, taking into account the implicit assumption about the information channel tell. The essential point is that the protocol works provided that only the firewall can write on tell. This can be formalised by proving the equivalence between the two systems relative to a restricted environment, one which does not allow write access to tell.

First some notation. Let us write $\Gamma \vdash_{k}^{\max } V: \mathrm{T}$ to mean

$-\Gamma r_{k} V: \mathrm{T}$

$-\Gamma \vdash_{k} V: \mathrm{T}^{\prime}$ implies $\mathrm{T}<: \mathrm{T}^{\prime}$

In other words, $\mathrm{T}$ is the largest type which can be assigned to $V$. Now suppose $\mathcal{I}$ is a type environment which satisfies

(i) $\mathcal{I} r_{a}^{\max }$ tell $: \mathrm{r}\langle\mathrm{F}\rangle$

(ii) $\mathcal{I} \vdash a \llbracket R \rrbracket$

(iii) $\mathcal{I} \vdash($ new $f:$ F $) f \llbracket P \rrbracket$

The import of the first requirement, which is the most important, is that systems in the computational context can not write on tell. The other requirements, which are mainly for convenience, ensure that the residual behaviour at $a$ and $f$ is well-behaved, although a side-effect is that they also can not write on tell. Under these assumptions, we prove

$$
\mathcal{I} \models F \mid A \approx_{b i s}(\text { new } f: \mathrm{F})(f \llbracket P \mid * \text { goto } a \text {.tell! }\langle f\rangle \mid Q \rrbracket) \mid a \llbracket R \rrbracket
$$

First note that (up-to structural equivalence)

$$
\mathcal{I} \triangleright F|A \stackrel{\tau}{\longrightarrow} F| A_{t} \mid a \llbracket R \rrbracket
$$

via (M-SPLIT) and (M-CTXT), where we use $A_{t}$ as a shorthand for $a \llbracket$ tell? $(x)$ goto $x . Q \rrbracket$. So, by Propositions 1 and 2, it is sufficient to prove

$$
\mathcal{I} \models F\left|A_{t}\right| a \llbracket R \rrbracket \approx_{b i s}(\text { new } f: \mathrm{F})(f \llbracket P \mid * \text { goto } a . \text { tell }\lfloor\langle f\rangle \mid Q \rrbracket) \mid a \llbracket R \rrbracket
$$

Here assumption (ii) comes in useful, as by Contextuality it is now sufficient to prove

$$
\mathcal{I} \models F \mid A_{t} \approx_{b i s}(\text { new } f: \mathrm{F})(f \llbracket P \mid * \text { goto } a \text {.tell! }\langle f\rangle \mid Q \rrbracket)
$$

Then the left-hand side can be manipulated via the structural equivalence rule (S-EXTR), thereby reducing the proof burden to

$$
\mathcal{I}=(\text { new } f: \mathrm{F})\left(f \llbracket P \mid * \text { goto } a \text {.tell! }\langle f\rangle \rrbracket \mid A_{t}\right) \approx_{b i s}(\text { new } f: \mathrm{F})(f \llbracket P \mid * \text { goto } a \text {.tell! }\langle f\rangle \mid Q \rrbracket)
$$

and another application of Contextuality reduces this further to

$$
\mathcal{I}_{f}=f \llbracket P \mid * \text { goto } a . \text { tell! }\langle f\rangle \rrbracket\left|A_{t} \approx_{b i s} f \llbracket P\right| * \text { goto a.tell! }\langle f\rangle \mid Q \rrbracket
$$

where $\mathcal{I}_{f}$ is a shorthand for $\mathcal{I},\langle f: \mathrm{F}\rangle$. Now let $F_{g}$ represent $f \llbracket *$ goto $a$.tell! $\langle f\rangle \rrbracket$. Then we have 
- $I_{f} \triangleright f \llbracket P \mid *$ goto $a$.tell! $\langle f\rangle \rrbracket\left|A_{t} \stackrel{\tau}{\longrightarrow}_{\beta} f \llbracket P \rrbracket\right| F_{g} \mid A_{t}$

- $\mathcal{I}_{f} \triangleright f \llbracket P \mid *$ goto $a$.tell! $\langle f\rangle\left|Q \rrbracket \stackrel{\tau}{\longrightarrow}{ }_{\beta}^{*} f \llbracket P \rrbracket\right| F_{g} \mid f \llbracket Q \rrbracket$

So, further applications of Proposition 2, Contextuality and assumption (iii), give the requirement

$$
\mathcal{I}_{f} \vDash F_{g}\left|A_{t} \approx_{b i s} \quad F_{g}\right| f \llbracket Q \rrbracket
$$

This we establish directly by exhibiting a particular bisimulation.

We define the parameterised relation $\mathcal{R}$ by letting $\mathcal{J} \vDash M \mathcal{R} N$ whenever

(a) $\mathcal{J} \triangleright M$ is a configuration and $N$ is the same as $M$

(b) or $\mathcal{J}$ is $\mathcal{I}_{f}$ and

- $M$ has the form $F_{g}\left|A_{t}\right| \Pi_{n}(a \llbracket \text { tell! }\langle f\rangle \rrbracket)^{n}$

- $N$ has the form $F_{g}|f \llbracket Q \rrbracket| \Pi_{n}(a \llbracket \text { tell! }\langle f\rangle \rrbracket)^{n}$

where $\Pi_{n}(a \llbracket \text { tell! }\langle f\rangle \rrbracket)^{n}$, for some $n \geq 0$, means $n$ copies of $a \llbracket$ tell! $\langle f\rangle \rrbracket$ running in parallel.

Proposition 4. The relation $\mathcal{R}$ defined above is a bisimulation up-to- $\beta$.

Proof. Suppose $\mathcal{J} \vDash M \mathcal{R} N$. Let us consider all possible actions from $\mathcal{J} \triangleright M$. In fact, it is sufficient to consider the case (b) above, when $\mathcal{J}$ and $M$ and $N$ are of the prescribed form. The actions fall into one of three categories (for convenience we shorten $\Pi_{n}(a \llbracket \text { tell! }\langle f\rangle \rrbracket)^{n}$ with $\left.\Pi_{n}\right)$.

- Here $F_{g}$ is responsible, so the action takes the form $\mathcal{I}_{f} \triangleright M \stackrel{\tau}{\longrightarrow} \beta[\llbracket *$ goto $a$.tell! $\langle f\rangle \mid$ goto $a$.tell! $\langle f\rangle \rrbracket\left|A_{t}\right| \Pi_{n}$. But $\mathcal{I}_{f} \triangleright f \llbracket *$ goto $a$.tell! $\langle f\rangle \mid$ goto $a$.tell! $\langle f\rangle \rrbracket\left|A_{t}\right| \Pi_{n} \stackrel{\tau}{\longrightarrow} \beta$ $F_{g} \mid a \llbracket$ tell! $\langle f\rangle \rrbracket\left|A_{t}\right| \Pi_{n}$, and this can be matched, via clause (b), by $\mathcal{I}_{f} \triangleright N \stackrel{\tau}{\longrightarrow} \beta$ $F_{g} \mid a \llbracket$ tell! $\langle f\rangle \rrbracket|f \llbracket Q \rrbracket| \Pi_{n}$, because $F_{g} \mid a \llbracket$ tell! $\langle f\rangle \rrbracket\left|A_{t}\right| \Pi_{n} \equiv F_{g}\left|A_{t}\right| \Pi_{n+1}$ and $F_{g} \mid a \llbracket$ tell! $\langle f\rangle \rrbracket|f \llbracket Q \rrbracket| \Pi_{n} \equiv F_{g}|f \llbracket Q \rrbracket| \Pi_{n+1}$ and $\equiv \subseteq \approx_{b i s}$ (Proposition 1).

- The second possibility is that the third component, $\Pi_{n}(a \llbracket \text { tell! }\langle f\rangle \rrbracket)^{n}$, is responsible for the action, which must be $a$.tell! $f$. It is easy to see that $\mathcal{I}_{f} \triangleright N$ can perform exactly the same action, to a related configuration in clause (b).

- Finally, the middle component, $A_{t}$, might be involved in the action. Note that the action can not be external, as the action $a$.tell? $V$ (for some value $V$ ) is not allowed by the environment. So it must be a communication, of the form $\mathcal{I}_{f} \triangleright M \stackrel{\tau}{\longrightarrow} F_{g} \mid$ $a \llbracket$ goto $f . Q \rrbracket \mid \Pi_{n-1}$. But the following $\beta$-steps can be carried out starting from this configuration: $\mathcal{I}_{f} \triangleright F_{g} \mid a \llbracket$ goto $f . Q \rrbracket\left|\Pi_{n-1} \stackrel{\tau}{\longrightarrow}{ }_{\beta}^{*} F_{g}\right| a \llbracket$ tell! $\langle f\rangle \rrbracket|f \llbracket Q \rrbracket| \Pi_{n-1} \equiv$ $F_{g}|f \llbracket Q \rrbracket| \Pi_{n}$, and this can be matched in clause (a) by the empty sequence of internal actions from $\mathcal{I}_{f} \triangleright N$.

Symmetrically, it is easy to see that every action from $\mathcal{J} \triangleright N$ can be matched by one from $\mathcal{J} \triangleright M$, possibly preceded by a number of $\tau$-actions: these latter are required when $f \llbracket Q \rrbracket$ is responsible for the action to be matched.

This, by using Proposition 3, completes our proof of (5) above. 
Note that the firewall $F$ allows, in principle, multiple entries of agents from $a$. So, for example, if $R$, in (5), had the form $R^{\prime} \mid$ tell?(x) goto $x . Q^{\prime}$, then the reasoning we have just completed could be repeated, to prove

$$
\left.\mathcal{I} \vDash F \mid a \llbracket R \rrbracket \approx_{b i s}(\text { new } f: \mathrm{F})(f \llbracket P \mid * \text { goto } a \text {.tell!!f }\rangle \mid Q^{\prime} \rrbracket\right) \mid a \llbracket R^{\prime} \rrbracket
$$

Moreover, we know $f$ can not appear in $Q$; therefore, (S-EXTR) from Figure 5 together with (7) can be combined with (5), to prove

$$
\mathcal{I} \vDash F \mid A \approx_{b i s}(\text { new } f: \mathrm{F})\left(f \llbracket P \mid * \text { goto } a \text {.tell! }\langle f\rangle|Q| Q^{\prime} \rrbracket\right) \mid a \llbracket R^{\prime} \rrbracket
$$

where the domain $a$ has managed to send two separate agents into the firewall.

\section{A Server and Its Clients}

We consider in this section the canonical example of a server and its clients. A server is a domain providing services to potentially arbitrary clients, as e.g. the following

$$
S \Leftarrow s \llbracket * \text { req? }(x, y \Subset z) \text { goto } z . y !\langle i \operatorname{sprime}(x)\rangle \mid S^{\prime} \rrbracket
$$

which provides an iterated service at resource req, and internal code, $S^{\prime}$, to setup and administrate the site. The channel req expects to receive a structured value of the form $\left(i, c_{\circledast} l\right)$. This is a pair, consisting of an integer $i$, and a return address $c_{\circledast} l$, that is the name of a reply channel, $c$, together with the location of that channel, $l$. The server then executes the procedure isprime (-) on the incoming value, $i$, sends a process to the return site, and delivers the result on the return channel there. The procedure isprime is not directly part of the language, but one can easily imagine an extension supporting let expressions, in which case the body of the server would be better represented as *req? $(x, y @ z)$ let $b=i$ sprime $(x)$ in goto $z \cdot y !\langle b\rangle$, thereby emphasising that the procedure is executed at the server's site.

Typical clients of the server are domains taking the form

$$
C_{i} \Leftarrow c_{i} \llbracket\left(\text { newc } r: \text { R) goto } s \text {.req! }\left\langle v_{i}, r_{\circledast} c_{i}\right\rangle \mid C_{i}^{\prime} \rrbracket\right.
$$

These generate a private reply channel $r$ at the declaration type $\mathrm{R}=\mathrm{rw}\langle\mathbf{b o o l}\rangle$, and send a process to the server (whose address they need to know) asking for the primality of an integer; concurrently, the agent $C_{i}^{\prime}$ executes at the site.

As in the case of the firewall, the correctness of the protocol between the server $S$ and its clients $C_{i}$ depends on the proper management of the access to the request channel req: clients should only have write access, while the server only needs read access. So the correctness of the protocol can be expressed as an equivalence between two systems, relative to a restricted environment. Let $\mathcal{I}$ be a type environment satisfying

(i) $\mathcal{I} \vdash_{s}^{\max }$ req : w $\left\langle\right.$ int, $\left.\mathrm{w}\langle\mathbf{b o o l}\rangle_{\circledast} \mid \mathrm{loc}\right\rangle$

(ii) $\mathcal{I} \vdash s \llbracket S^{\prime} \rrbracket$

(iii) $\mathcal{I} \vdash C_{i}$ 
The first requirement establishes the computational context can not read on req, while the following points ensure that the residual behaviour at the server and the clients is well-behaved, with the side-effect that neither $S^{\prime}$ nor $C_{i}^{\prime}$ can read on req.

First, let us show that one client interacts correctly with the server

$$
\mathcal{I} \models S\left|C_{1} \approx_{b i s} S\right| c_{1} \llbracket(\text { newc } r: \mathrm{R}) r \text { ! }\left\langle i \operatorname{sprime}\left(v_{1}\right)\right\rangle \mid C_{1}^{\prime} \rrbracket
$$

Note that (up-to-structural equivalence)

$$
\mathcal{I} \triangleright S \mid C_{1} \stackrel{\tau}{\longrightarrow}{ }_{\beta}^{*}\left(\text { new } r @ c_{1}: \mathrm{R}\right) S_{r}\left|s \llbracket S^{\prime} \rrbracket\right| s \llbracket \text { req! }\left\langle v_{1}, r_{\circledast} c_{1}\right\rangle \rrbracket \mid c_{1} \llbracket C_{1}^{\prime} \rrbracket
$$

where we use $S_{r}$ as a shorthand for $s \llbracket *$ req? $(x, y @ z)$ goto $z . y !\langle i \operatorname{sprime}(x)\rangle \rrbracket$, and

$$
\begin{aligned}
& \mathcal{I} \triangleright S \mid c_{1} \llbracket(\text { newc } r: \mathrm{R}) r !\left\langle i \operatorname{sprime}\left(v_{1}\right)\right\rangle \mid C_{1}^{\prime} \rrbracket \stackrel{\tau}{\longrightarrow}{ }_{\beta}^{*} \\
& \quad\left(\text { new } r_{\circledast} c_{1}: \mathrm{R}\right) S_{r}\left|s \llbracket S^{\prime} \rrbracket\right| c_{1} \llbracket r !\left\langle i \operatorname{sprime}\left(v_{1}\right)\right\rangle \rrbracket \mid c_{1} \llbracket C_{1}^{\prime} \rrbracket
\end{aligned}
$$

By Propositions 1, 2, Contextuality, and assumptions (ii), (iii), it is therefore sufficient to prove

$$
\mathcal{I}_{r} \vDash S_{r}\left|s \llbracket \mathrm{req} !\left\langle v_{1}, r_{\Theta} c_{1}\right\rangle \rrbracket \approx_{b i s} S_{r}\right| c_{1} \llbracket r !\left\langle i \operatorname{sprime}\left(v_{1}\right)\right\rangle \rrbracket
$$

where $\mathcal{I}_{r}$ is a shorthand for $\mathcal{I},\left\langle r_{\circledast} c_{1}: \mathrm{R}\right\rangle$. We establish this equivalence by exhibiting a particular bisimulation. Let $\mathcal{R}$ be the parameterised relation defined by letting $\mathcal{J} \vDash M \mathcal{R}$ $N$ whenever

(a) $\mathcal{J} \triangleright M$ is a configuration and $N$ is the same as $M$

(b) or $\mathcal{J}$ is $\mathcal{I}_{r}$ and

- $M$ has the form $S_{r} \mid s \llbracket$ req! $\left\langle v_{1}, r_{\Theta} c_{1}\right\rangle \rrbracket \mid \Pi_{n}$

- $N$ has the form $S_{r}\left|c_{1} \llbracket r !\left\langle i \operatorname{sprime}\left(v_{1}\right)\right\rangle \rrbracket\right| \Pi_{n}$

where $\Pi_{n}$ is a shorthand for $\Pi_{n}\left(s \llbracket \text { req? }\left(x, y_{@} z\right) \text { goto } z . y !\langle i \operatorname{sprime}(x)\rangle \rrbracket\right)^{n}$

(c) or $\mathcal{J}$ is $\mathcal{I}_{r}^{\prime}$, where the domain of $\mathcal{I}_{r}^{\prime}$ is a superset of that of $\mathcal{I}_{r}$, and

- $M$ has the form $S_{r} \mid s \llbracket$ req! $\left\langle v_{1}, r_{\Theta} c_{1}\right\rangle \rrbracket\left|\Pi_{n}\right| \Pi_{j \in J}\left(k_{j} \llbracket d_{j} !\left\langle i \operatorname{isprime}\left(i_{j}\right)\right\rangle \rrbracket\right)$

- $N$ has the form $S_{r}\left|c_{1} \llbracket r !\left\langle i \operatorname{sprime}\left(v_{1}\right)\right\rangle \rrbracket\right| \Pi_{n} \mid \Pi_{j \in J}\left(k_{j} \llbracket d_{j} !\left\langle i \operatorname{sprime}\left(i_{j}\right)\right\rangle \rrbracket\right)$

such that $\mathcal{I}_{r}^{\prime} r_{s}^{\max }$ req:w $\left\langle\right.$ int, $\left.\mathbf{w}\langle\mathbf{b o o l}\rangle_{\Theta} \mid \mathbf{l o c}\right\rangle$, and, for every $j \in J: I_{r}^{\prime} \vdash_{k_{j}} d_{j}: \mathrm{w}\langle\mathbf{b o o l}\rangle$. The notation $\Pi_{j \in J}\left(k_{j} \llbracket d_{j} !\left\langle i\right.\right.$ isprime $\left.\left.\left(i_{j}\right)\right\rangle \rrbracket\right)$ means (different) instances of systems running in parallel.

Proposition 5. The relation $\mathcal{R}$ defined above is a bisimulation up-to- $\beta$.

Proof. Suppose $\mathcal{J} \vDash M \mathcal{R} N$. The actions from $\mathcal{J} \triangleright M$ in the case (b) above fall into one of three categories.

- First $S_{r}$ is responsible: $\mathcal{I}_{r} \triangleright M \stackrel{\tau}{\longrightarrow}{ }_{\beta}^{*} S_{r}\left|\Pi_{1}\right| s \llbracket$ req! $\left\langle v_{1}, r_{\circledast} c_{1}\right\rangle \rrbracket \mid \Pi_{n}$, and this can be matched by $\mathcal{I}_{r} \triangleright N \stackrel{\tau}{\longrightarrow}{ }_{\beta}^{*} S_{r}\left|\Pi_{1}\right| c_{1} \llbracket r !\left\langle i \operatorname{isprime}\left(v_{1}\right)\right\rangle \rrbracket \mid \Pi_{n}$, because both configurations belong to $\mathcal{R}$, clause (b), up-to structural equivalence. 
- The third component, $\Pi_{n}(s \llbracket \text { req? }(x, y \Subset z) \text { goto } z . y !\langle i \operatorname{sprime}(x)\rangle \rrbracket)^{n}$, is responsible for the action, which is either $s . r e q ?\left\langle i_{j}, d_{j} @ k_{j}\right\rangle$ or $(e: E) s . r e q ?\left\langle i_{j}, d_{j} @ k_{j}\right\rangle$. These actions correspond to the delivery of (new) data by the environment (from which the system is allowed to learn infinitely new names), and are followed by the action (M-MOVE). However, it is easy to see that $\mathcal{I}_{r} \triangleright N$ can perform exactly the same actions, to a related configuration in clause (c).

- Finally, the middle component, $s \llbracket$ req! $\left\langle v_{1}, r_{\circledast} c_{1}\right\rangle \rrbracket$, may be involved in the action, which must be a communication: $\mathcal{I}_{r} \triangleright M \stackrel{\tau}{\longrightarrow} S_{r} \mid s \llbracket$ goto $c_{1} . r !\left\langle i \operatorname{sprime}\left(v_{1}\right)\right\rangle \rrbracket \mid \Pi_{n-1}$. Then the following $\beta$-steps can be carried out: $\mathcal{I}_{r} \triangleright S_{r} \mid s \llbracket$ goto $c_{1} . r !\left\langle\right.$ isprime $\left.\left(v_{1}\right)\right\rangle \rrbracket \mid$ $\Pi_{n-1} \stackrel{\tau}{\longrightarrow}{ }_{\beta}^{*} S_{r}\left|\Pi_{1}\right| c_{1} \llbracket r !\left\langle i\right.$ sprime $\left.\left(v_{1}\right)\right\rangle \rrbracket \mid \Pi_{n-1}$, and this configuration can be matched, in clause (a), by the empty sequence of actions from $\mathcal{I}_{r} \triangleright N$.

Symmetrically, every action performed by $\mathcal{I}_{r} \triangleright N$ can be matched by $\mathcal{I}_{r} \triangleright M$; for example, consider the output action by the 2nd component of $N: \mathcal{I}_{r} \triangleright S_{r} \mid c_{1} \llbracket\left[r !\left\langle i s p r i m e\left(v_{1}\right)\right\rangle \rrbracket \mid\right.$ $\Pi_{n} \stackrel{c_{1} . r\left\{i s p r i m e\left(v_{1}\right)\right\rangle}{\longrightarrow} \mathcal{I}_{r} \triangleright S_{r} \mid \Pi_{n}$. This can be easily matched by $\mathcal{I}_{r} \triangleright M$, via clause (a), using $\tau$-steps followed by the same action.

Finally, it is not problematic to check that all configurations in $\mathcal{R}$ by virtue of clause (c) can have their respective actions properly matched.

This completes our proof of (8), that one client can interact correctly with the server. Contextual reasoning can now be employed to generalise this result to an arbitrary number of clients. For example, let us show

$$
\mathcal{I} \vDash S\left|C_{1}\right| C_{2} \approx_{b i s} S \mid \Pi_{i \in\{1,2\}} c_{i} \llbracket(\text { newc } r: \mathrm{R}) r !\left\langle i \operatorname{sprime}\left(v_{i}\right)\right\rangle \mid C_{i}^{\prime} \rrbracket
$$

Because of $\mathcal{I} \vdash C_{2}$ (requirement (iii) above), Contextuality applied to (8) gives

$$
\mathcal{I} \models S\left|C_{1}\right| C_{2} \approx_{b i s} S \mid c_{1} \llbracket(\text { newc } r: \mathrm{R}) r !\left\langle i \operatorname{sprime}\left(v_{1}\right)\right\rangle\left|C_{1}^{\prime} \rrbracket\right| C_{2}
$$

Repeating the analysis of $C_{1}$ on $C_{2}$, we obtain

$$
\mathcal{I} \models S\left|C_{2} \approx_{b i s} S\right| c_{2} \llbracket(\text { newc } r: \mathrm{R}) r \text { ! }\left\langle\text { isprime }\left(v_{2}\right)\right\rangle \mid C_{2}^{\prime} \rrbracket
$$

But $\mathcal{I} \vdash C_{1}$ (same requirement (iii)) also implies $\mathcal{I} \vdash c_{1} \llbracket($ newc $r: \mathrm{R}) r$ ! $\left\langle\right.$ isprime $\left.\left(v_{1}\right)\right\rangle \mid$ $C_{1}^{\prime} \rrbracket$, and therefore by Contextuality we obtain

$$
\begin{aligned}
\mathcal{I}= & S\left|C_{2}\right| c_{1} \llbracket(\text { newc } r: \mathrm{R}) r !\left\langle i \text { sprime }\left(v_{1}\right)\right\rangle \mid C_{1}^{\prime} \rrbracket \approx_{b i s} \\
& S \mid c_{2} \llbracket(\text { newc } r: \mathrm{R}) r !\left\langle i \operatorname{sprime}\left(v_{2}\right)\right\rangle\left|C_{2}^{\prime} \rrbracket\right| c_{1} \llbracket(\text { newc } r: \mathrm{R}) r !\left\langle i \operatorname{sprime}\left(v_{1}\right)\right\rangle \mid C_{1}^{\prime} \rrbracket
\end{aligned}
$$

So we conclude (9) from (10), Proposition 1 , and transitivity of $\approx_{b i s}$.

It is then a simple matter to extend this reasoning, using induction, to show that an arbitrary number of clients can be handled

$$
\mathcal{I} \models S\left|\Pi_{i \in\{1, \ldots, n\}} C_{i} \approx_{b i s} S\right| \Pi_{i \in\{1, \ldots, n\}} c_{i} \llbracket(\text { newc } r: \mathrm{R}) r !\left\langle i \operatorname{sprime}\left(v_{i}\right)\right\rangle \mid C_{i}^{\prime} \rrbracket
$$

\section{Metaservers}

In this section we describe a memory service by involving the newloc operator of Dir, which allows the creation of new instances of sites. A (meta)server contains a resource 
setup, where requests are received, and installs the service at a new site, thus providing personalised treatment to its clients.

A first version of the server receives a return address, generates a new located memory cell, and installs some code there, meanwhile delivering the new location name at the reply address

$$
S \Leftarrow s \llbracket * \operatorname{setup} \text { ?(y@z) (newloc } m: \text { M) goto } m . \text { Mem | goto } z . y !\langle m\rangle \rrbracket
$$

where Mem is the code running at the location $m$, and for instance can take the form

$$
\begin{aligned}
& \text { Mem } \Leftarrow(\text { newc } v: \mathrm{V}) v !\langle 0\rangle \mid * \operatorname{get} ?\left(y_{\odot} z\right) v ?(w)(\text { goto } z \cdot y !\langle w\rangle \mid v !\langle w\rangle) \\
& \mid \text { *put?(x,y@z) } v ?(w) \text { (goto } z . y ! \mid v !\langle x\rangle)
\end{aligned}
$$

Here we are using the channel $v$ as a restricted form of memory cell: the value it contains (whose initial value is set to 0 ) disappears once it is read, therefore it has to be reinstated. The two methods get and put can be seen as the canonical ways to access the cell, therefore the declaration type of the new site can be set to $M=\operatorname{loc}\left[\right.$ get : $T_{g}$, put : $\mathrm{T}_{p}$ ]. Notice that we have chosen this particular instantiation for the running code Mem just for reasons of simplicity, as the proofs we are going to develop are, in principle, independent of it.

Clients of the memory service generate a new reply channel, send a request to the server, and wait for the server to deliver the new memory cell

$$
C_{i} \Leftarrow c_{i} \llbracket(\text { newc } r: \mathrm{R}) \text { goto } s . \operatorname{setup} !\left\langle r_{\Theta} c_{i}\right\rangle \mid r ?(x) P_{i}(x) \rrbracket
$$

where $P_{i}(x)$ is parametric code which depends on (the name of) the new site, $x$, and $\mathrm{R}=\mathrm{rw}\langle\mathrm{M}\rangle$.

An alternative, slightly different version of the server leaves to the clients the responsibility to create the memory cells, just installing the servicing code at the proffered site

$$
S^{\prime} \Leftarrow s^{\prime} \llbracket * \operatorname{setup}^{\prime} ?\left(x, y_{\oplus} z\right) \text { goto } x \text {.Mem | goto } z . y \text { ! }
$$

Correspondingly, clients generate an acknowledgement channel and a new location, send a request to the server, and await the server to acknowledge the service has been installed

$$
C_{i}^{\prime} \Leftarrow c_{i} \llbracket(\text { newc } t: \mathrm{T})\left(\text { newloc } m_{i}: \mathrm{M}\right) \text { goto } s^{\prime} \cdot \operatorname{setup}^{\prime} !\left\langle m_{i}, t @ c_{i}\right\rangle \mid t ? P_{i}\left(m_{i}\right) \rrbracket
$$

where $\mathrm{T}=\mathrm{rw}\langle\mathbf{u n i t}\rangle$.

We want now to relate the two different approaches, therefore connecting the behaviour of the two following systems, relative to a typing environment $\mathcal{I}$

$$
\begin{aligned}
& \mathcal{I} \models S\left|C_{1}\right| C_{2} \\
& \mathcal{I} \models S^{\prime}\left|C_{1}^{\prime}\right| C_{2}^{\prime}
\end{aligned}
$$

Our goal is to establish that, from the point of view of the clients, under certain hypotheses the two kinds of servers $S$ and $S^{\prime}$ lead to equivalent behaviour. This means finding a suitable type environment $\mathcal{I}$ such that

$$
\mathcal{I} \models S\left|C_{1}\right| C_{2} \approx_{b i s} S^{\prime}\left|C_{1}^{\prime}\right| C_{2}^{\prime}
$$


It is immediate to notice that the correctness of this protocol requires that the computational context should have neither write nor read access to the setup and setup' channels. Thus, the equivalence can be proved relative to a restricted environment $\mathcal{I}$, satisfying

$$
\mathcal{I} r_{s}^{\max } \text { setup : } \top \quad \mathcal{I} r_{s^{\prime}}^{\max } \text { setup }^{\prime}: \top
$$

Now, the internal actions allow to deduce a derivation from (11) and (12) to the systems

$$
\begin{aligned}
& \mathcal{I} \models S \mid \Pi_{i \in\{1,2\}}\left(\text { new } m_{i}: \mathrm{M}\right)\left(m_{i} \llbracket \text { Mem } \rrbracket \mid c_{i} \llbracket P_{i}\left(m_{i}\right) \rrbracket\right) \\
& \mathcal{I} \vDash S^{\prime} \mid \Pi_{i \in\{1,2\}}\left(\text { new } m_{i}: \mathrm{M}\right)\left(m_{i} \llbracket \text { Mem } \rrbracket \mid c_{i} \llbracket P_{i}\left(m_{i}\right) \rrbracket\right)
\end{aligned}
$$

Therefore we address (13) in three steps: first we prove that the two pairs of systems (11),(14) and (12),(15) are equivalent, then we connect the systems (14) and (15) by a technical lemma. That is

(i) $\mathcal{I} \models S\left|\Pi_{i \in\{1,2\}} C_{i} \approx_{b i s} S\right| \Pi_{i \in\{1,2\}}\left(\right.$ new $\left.m_{i}: \mathrm{M}\right)\left(m_{i} \llbracket \mathrm{Mem} \rrbracket \mid c_{i} \llbracket P_{i}\left(m_{i}\right) \rrbracket\right)$

(ii) $\mathcal{I} \models S^{\prime}\left|\Pi_{i \in\{1,2\}} C_{i}^{\prime} \approx_{b i s} S^{\prime}\right| \Pi_{i \in\{1,2\}}\left(\right.$ new $\left.m_{i}: \mathrm{M}\right)\left(m_{i} \llbracket \mathrm{Mem} \rrbracket \mid c_{i} \llbracket P_{i}\left(m_{i}\right) \rrbracket\right)$

(iii) $\mathcal{I} \models l \llbracket * a ?(x) P(x) \rrbracket \mid Q \approx_{b i s} Q$ for every $\mathcal{I}, Q, l, a, P$ s. t. $\mathcal{I} \vdash_{l}^{\max } a$ : T and $a \notin \mathrm{fn}(Q)$

The proof of the point (iii) is straightforward, as a witness bisimulation $\mathcal{R}$ can be promptly defined by letting $\mathcal{J} \vDash M \mathcal{R} N$ whenever

(a) $\mathcal{T} \triangleright M$ and $\mathcal{J} \triangleright N$ are configurations

(b) $\mathcal{J}$ is $\mathcal{I}$ and $M$ has the form $l \llbracket * a ?(x) P(x) \rrbracket\left|\Pi_{n}(l \llbracket a ?(x) P(x) \rrbracket)^{n}\right| N$

which can be easily proved to be a bisimulation up-to- $\beta$.

We argue below both the proof of (i) (the one of (ii) is completely similar) and how to get the proof of (13) from those of (i), (ii), (iii). Let us start from the latter.

Using the equations (i) and (ii), the equivalence (13) can be reduced to

$$
\mathcal{I} \models S\left|\Pi_{i \in\{1,2\}} Q_{i} \approx_{b i s} S^{\prime}\right| \Pi_{i \in\{1,2\}} Q_{i}
$$

where $Q_{i}$ denotes (new $\left.m_{i}: \mathrm{M}\right)\left(m_{i} \llbracket \mathrm{Mem} \rrbracket \mid c_{i} \llbracket P_{i}\left(m_{i}\right) \rrbracket\right)$. It is natural now to assume that the conditions required by the lemma (iii) are satisfied by the code $Q_{i}$ (setup, setup' $\notin$ $\mathrm{fn}\left(Q_{i}\right)$, in the case). Hence, it is possible to apply that lemma to both the sides of the equation (16), thus obtaining an identity.

Finally, we address the point (i). First notice that (up-to structural equivalence)

$$
\begin{aligned}
& \mathcal{I} \triangleright S \mid \Pi_{i \in\{1,2\}} C_{i} \stackrel{\tau}{\longrightarrow} * \\
& \text { (new } \left.r_{1} \oplus c_{1}: \mathrm{R}, r_{2} \oplus c_{2}: \mathrm{R}\right) S \mid \Pi_{i \in\{1,2\}}\left(s \llbracket \text { setup! }\left\langle r_{i} \oplus c_{i}\right\rangle \rrbracket \mid c_{i} \llbracket r_{i} \text { ? }(x) P_{i}(x) \rrbracket\right)
\end{aligned}
$$

and $S \mid \Pi_{i \in\{1,2\}} Q_{i} \equiv$ (new $\left.m_{1}: \mathrm{M}, m_{2}: \mathrm{M}\right) S \mid \Pi_{i \in\{1,2\}}\left(m_{i} \llbracket \mathrm{Mem} \rrbracket \mid c_{i} \llbracket P_{i}\left(m_{i}\right) \rrbracket\right)$. Therefore, by Propositions 1, 2, we reduce (i) to the following

$$
\begin{aligned}
\mathcal{I} \vDash & \left(\text { new } r_{1} @ c_{1}: \mathrm{R}, r_{2} @ c_{2}: \mathrm{R}\right) S \mid \Pi_{i \in\{1,2\}}\left(s \llbracket \operatorname{setup} !\left\langle r_{i} @ c_{i}\right\rangle \rrbracket \mid c_{i} \llbracket r_{i} ?(x) P_{i}(x) \rrbracket\right) \approx_{b i s} \\
& \left(\text { new } m_{1}: \mathrm{M}, m_{2}: \mathrm{M}\right) S \mid \Pi_{i \in\{1,2\}}\left(m_{i} \llbracket \operatorname{Mem} \rrbracket \mid c_{i} \llbracket P_{i}\left(m_{i}\right) \rrbracket\right)
\end{aligned}
$$

which we prove by exhibiting a particular bisimulation. Let us fix before some shorthand notation

$$
\begin{aligned}
C_{! i} \triangleq c_{i} \llbracket r_{i} !\left\langle m_{i}\right\rangle \rrbracket & C_{? i} \triangleq c_{i} \llbracket r_{i} ?(x) P_{i}(x) \rrbracket \\
M_{i} \triangleq m_{i} \llbracket \text { Mem } \rrbracket & S_{! i} \triangleq s \llbracket \text { setup! }\left\langle r_{i} @ c_{i}\right\rangle \rrbracket \\
C_{P_{i}} \triangleq c_{i} \llbracket P_{i}\left(m_{i}\right) \rrbracket & B \triangleq\left(\text { new } m_{1}, m_{2}: \mathrm{M}\right) S\left|\Pi_{n}\right| M_{1}\left|C_{P_{1}}\right| M_{2} \mid C_{P_{2}}
\end{aligned}
$$


and $\Pi_{n} \triangleq \Pi_{n}(s \llbracket$ setup?(y@z) (newloc $m: \mathrm{M})$ goto $m$.Mem $\mid$ goto $\left.z . y !\langle m\rangle \rrbracket\right)^{n}$. We define the relation $\mathcal{R}$ by letting $\mathcal{J} \vDash P \mathcal{R} Q$ whenever $\mathcal{J} \triangleright P$ is a configuration and $Q$ is the same as $P$, or $\mathcal{J}$ is $\mathcal{I}$ and $Q$ has the form $B$ and $P$ has the form

(a) (new $\left.r_{1} @ c_{1}: \mathrm{R}, r_{2} @ c_{2}: \mathrm{R}\right) S\left|\Pi_{n}\right| S_{! 1}\left|S_{! 2}\right| C_{? 1} \mid C_{? 2}$

(b) or (new $\left.r_{1} @ c_{1}: \mathrm{R}, r_{2} @ c_{2}: \mathrm{R}, m_{1}: \mathrm{M}\right) S\left|\Pi_{n}\right| S_{! 2}\left|C_{? 2}\right| M_{1}\left|C_{! 1}\right| C_{? 1}$

(c) or (new $\left.r_{1} @ c_{1}: \mathrm{R}, r_{2} @ c_{2}: \mathrm{R}, m_{2}: \mathrm{M}\right) S\left|\Pi_{n}\right| S_{! 1}\left|C_{? 1}\right| M_{2}\left|C_{! 2}\right| C_{? 2}$

(d) or (new $\left.r_{2} @ c_{2}: \mathrm{R}, m_{1}: \mathrm{M}\right) S\left|\Pi_{n}\right| S_{! 2}\left|C_{? 2}\right| M_{1} \mid C_{P_{1}}$

(e) or (new $\left.r_{1} @ c_{1}: \mathrm{R}, m_{2}: \mathrm{M}\right) S\left|\Pi_{n}\right| S_{! 1}\left|C_{? 1}\right| M_{2} \mid C_{P_{2}}$

(f) or (new $\left.r_{1} @ c_{1}: \mathrm{R}, r_{2} @ c_{2}: \mathrm{R}, m_{1}: \mathrm{M}, m_{2}: \mathrm{M}\right) S\left|\Pi_{n}\right| M_{1}\left|C_{! 1}\right| C_{? 1}\left|M_{2}\right| C_{! 2} \mid C_{? 2}$

(g) or (new $\left.r_{2} @ c_{2}: \mathrm{R}, m_{1}: \mathrm{M}, m_{2}: \mathrm{M}\right) S\left|\Pi_{n}\right| M_{1}\left|C_{P_{1}}\right| M_{2}\left|C_{! 2}\right| C_{? 2}$

(h) or (new $\left.r_{1} @ c_{1}: \mathrm{R}, m_{1}: \mathrm{M}, m_{2}: \mathrm{M}\right) S\left|\Pi_{n}\right| M_{1}\left|C_{! 1}\right| C_{? 1}\left|M_{2}\right| C_{P_{2}}$

Proposition 6. The relation $\mathcal{R}$ defined above is a bisimulation up-to- $\beta$.

We omit the proof of this Proposition, as no extra critical aspects arise with respect to the proofs detailed in the previous two sections. Summing up, we have shown (13) under the following assumptions

- $\mathcal{I} r_{s}^{\max }$ setup : $\top$ and $\mathcal{I} r_{s^{\prime}}^{\max } \operatorname{setup}^{\prime}: T$

- setup $\notin$ fn(Mem) and setup' $\notin$ fn(Mem)

- setup $\notin \mathrm{fn}\left(P_{i}\right)$ and setup' $\notin \mathrm{fn}\left(P_{i}\right)$

It is then possible to consider an arbitrary number of clients. The correctness of these can once more be addressed using the techniques, such as Contextuality, discussed in the previous sections.

\section{Related and Future Work}

Proofs of correctness of protocols or language translations are often carried out with respect to contextual equivalences [GC99,LS00]. Nevertheless, the use of bisimulationbased notions of equivalences enables such proofs to be considerably simplified. For instance, in [MN03], two up-to proof techniques (up-to expansion and up-to context) are borrowed from the PICALCULus and adapted to develop an algebraic theory and prove the correctness of the perfect firewall protocol [CG98]. Our paper tries to contribute to this second approach, using bisimulations, extending their application to situations in which the environment plays a significant role in system behaviour.

In this document, we have defined and illustrated a collection of methods for proving bisimulation equivalences for distributed, mobile systems, modelled with the DPI calculus [HR02b]. In order to cope with bisimulation equivalence in DPI [HMR04], it is natural to look for bisimulations up-to in the spirit of [SM92]. More precisely, we have introduced in our work bisimulations up-to $\beta$-reductions, which have been inspired by a similar approach to concurrent ML [JR04]. This technique relieves the burden of exhibiting witness bisimulations, and its feasibility has been proved to be successful, combined with Contextuality, for addressing the verification of sample access protocols, such as crossing a firewall, the interaction between a server and its clients, and metaservers providing memory services. 
In the future, we plan to test further with the up-to $\beta$-reduction technique we have devised, by dealing with more involved protocols, possibly in the spirit of [US01]. That work uses a novel notion of coupled simulation that, despite not coinciding with any contextual equivalence, allows the proof of correctness of a simple central-forwardingserver algorithm.

We would like also to extend the results and techniques stated for DPI to the more involved SAFEDPI [HRY04], which takes into account extra safety aspects of distributed systems.

Acknowledgements. The authors would like to acknowledge the financial support of the two EU Global Computing projects, Mikado and Myths.

\section{References}

[CG98] Luca Cardelli and Andrew D. Gordon. Mobile ambients. In Proc. of FoSSaCS, Lecture Notes in Computer Science 1378, Springer, 1998.

[CHR05] Alberto Ciaffaglione, Matthew Hennessy, and Julian Rathke. Proof methodologies for behavioural equivalence in DPI. Technical Report 03:2005, Department of Informatics, University of Sussex, http: //www. dimi.uniud.it/ ciaffagl, 2005.

[GC99] Andrew D. Gordon and Luca Cardelli. Equational properties of mobile ambients. In Proc. of FoSSaCS, Lecture Notes in Computer Science 1578, Springer, 1999.

[HMR04] Matthew Hennessy, Massimo Merro, and Julian Rathke. Towards a behavioural theory of access and mobility control in distributed systems. Th. Comp. Science 322(3), 2004.

[HR02a] Matthew Hennessy and Julian Rathke. Typed behavioural equivalences for processes in the presence of subtyping. Electronic Notes in Th. Comp. Science 61, 2002.

[HR02b] Matthew Hennessy and James Riely. Resource access control in systems of mobile agents. Information and Computation 173(1), 2002.

[HRY04] Matthew Hennessy, Julian Rathke, and Nobuko Yoshida. SAFEDPI: a language for controlling mobile code. In Proc. of FoSSaCS, LNCS 2987, Springer, 2004.

[JR04] Alan Jeffrey and Julian Rathke. A theory of bisimulation for a fragment of concurrent ML with local names. Theoretical Computer Science 323(1-3), 2004.

[LS00] Francesca Levi and Davide Sangiorgi. Controlling interference in ambients. In Proc. of POPL, 2000.

[Mil89] Robin Milner. Communication and Concurrency. Prentice Hall, 1989.

[MN03] Massimo Merro and Francesco Zappa Nardelli. Bisimulation proof methods for mobile ambients. In Proc. of ICALP, Lecture Notes in Computer Science 2719, Springer, 2003.

[MPW92] Robin Milner, Joachim Parrow, and David Walker. A calculus of mobile processes (I and II). Information and Computation, 100(1,2), 1992.

[PS00] Benjamin C. Pierce and Davide Sangiorgi. Behavioral equivalence in the polymorphic PICALCULUS. Journal of ACM 47(3), 2000.

[SM92] Davide Sangiorgi and Robin Milner. The problem of "weak bisimulation up to". In Proc. of CONCUR, Lecture Notes in Computer Science 630, Springer, 1992.

[SW01] Davide Sangiorgi and David Walker. The PICALCULUS: a Theory of Mobile Processes. Cambridge University Press, 2001.

[US01] Asis Unyapoth and Peter Sewell. Nomadic pict: correct communication infrastructure for mobile computation. In Proc. of POPL, 2001. 